\title{
Uniform and individual convergence rates for convex density classes
}

\section{Alexander Meister}

Received: August 8, 2007; Accepted: April 24, 2008

Summary: In this paper, we prove coincidence of uniform and individual convergence rates for convex density classes, which are Baire spaces with respect to the $L_{1}\left(\mathbb{R}^{d}\right)$-metric, in the field of nonparametric estimation. We show that for convex density classes, which are no Baire spaces, the validity of this result is not guaranteed, in general.

\section{Introduction}

Studying the convergence rates of the risk has become a widely-used method for evaluating the asymptotic quality of an estimator for large sample sizes. That refers to the generic statistical model where one observes the i.i.d. data $X_{1}, \ldots, X_{n}$ having the probability density $f$. The goal is estimating the density $f$ or some characteristics of $f$, e.g. its derivative or some functional of $f$. Our consideration is restricted to those characteristics with a linear link to $f$; more precisely, we estimate $\Phi_{t}(f)$ where $\Phi_{t}$ denotes a known linear operator. The standard problem of density estimation, i.e. estimating $f$ itself, is included by putting $\Phi_{t}=\mathrm{Id}$, the identity operator. We include the multivariate case where the data are $\mathbb{R}^{d}$-valued $(d \geq 1)$. In order to measure the estimation error, $\Phi_{t}(f)$ shall be contained in a normed linear space $\left(T,\|\cdot\|_{T}\right)$. As some deterministic a-priori knowledge about $f$ is usually given, e.g. some nonparametric smoothness conditions, we assume that $f$ lies in a known set $\mathcal{F} \subseteq L_{1}\left(\mathbb{R}^{d}\right)$.

Further, we consider linear inverse problems where the observations are not drawn from density $f$ but $\Phi_{d}(f)$ with another known linear operator $\Phi_{d}$, mapping onto a linear space $D$ equipped with the $L_{1}\left(\mathbb{R}^{d}\right)$-norm. Also, we assume continuity of $\Phi_{d}$ with respect to the $L_{1}\left(\mathbb{R}^{d}\right)$-norm on both $\mathcal{F}$ and $D$; and that $\Phi_{d}(f)$, for any $f \in \mathcal{F}$, is a density function; while $\mathcal{F}$ still consists of density functions. As a famous example, we mention density deconvolution where the data are observed with independent additive measurement error with known density $g$. Then $\Phi_{d}$ becomes the convolution operator with density $g$. Linearity and continuity with the induced operator norm $\left\|\Phi_{d}\right\|_{\left(L_{1}\left(\mathbb{R}^{d}\right), L_{1}\left(\mathbb{R}^{d}\right)\right)} \leq 1$ can be verified. Again, the case of no contamination is embedded by setting $\Phi_{d}=$ Id. The linear 
space $F \subseteq L_{1}\left(\mathbb{R}^{d}\right)$, which contains $\mathcal{F}$ as a subset, is the joint domain of $\Phi_{t}$ and $\Phi_{d}$. In many cases, the linear hull of $\mathcal{F}$ in $L_{1}\left(\mathbb{R}^{d}\right)$ can be chosen to be $F$.

The estimator of $\Phi_{t}(f)$ is denoted by $\hat{f}_{n}=\hat{f}_{n}\left(X_{1}, \ldots, X_{n}\right)$. The estimator $\hat{f}_{n}$ is required to be in $T$ almost surely for all $f \in \mathcal{F}$. We define

$$
\Gamma_{n}(f):=a_{n}^{-1} E\left\|\hat{f}_{n}-\Phi_{t}(f)\right\|_{T}^{k}, \quad f \in \mathcal{F}
$$

with $k \geq 1$ and some positive-valued sequence $\left(a_{n}\right)_{n} \downarrow 0$. Then, $\left(a_{n}\right)_{n}$ represents the proposed convergence rate.

Estimator $\hat{f}_{n}$ is said to achieve the individual rate $\left(a_{n}\right)_{n}$ if

$$
\forall f \in \mathcal{F} \exists C(f)>0: \sup _{n \in \mathbb{N}} \Gamma_{n}(f) \leq C(f) .
$$

On the other hand, estimator $\hat{f}_{n}$ is said to achieve the uniform rate $\left(a_{n}\right)_{n}$ if

$$
\exists C>0: \sup _{n \in \mathbb{N}} \sup _{f \in \mathcal{F}} \Gamma_{n}(f) \leq C .
$$

Quite obviously, (1.1) follows from (1.2). However, the reverse implication is not valid in general. In terms of density estimation, research has mainly focused on minimax estimation, i.e. optimal uniform rates with respect to an arbitrary estimator, see Stone (1980), Donoho and Liu (1991a, b), Fan (1991, 1993), Korostelev and Tsybakov (1993), Yang and Barron (1999) among many others; while individual rates in density estimation are considered in Devroye (1983, 1995), Birgé (1986); the papers of Antos (2002) and Antos and Lugosi (1998) refer to a classification model in the field of individual rates; in Antos et al. (2000) individual rates are considered in a regression model.

Unlike previous research, which mainly concentrates on uniform and individual rates in specific settings, we intend to develop a general link between those two types of asymptotic views. In Brown et al. (1997), examples for everywhere superefficient estimators are given, implying non-coincidence of (1.1) and (1.2) in our notation. Barron and Hengartner (1998) give general results to reduce superefficiency to a measure-zero set with respect to the Hellinger and the Kullback-Leibler distance; in the nonparametric part of that work, assumptions on the covering numbers of the underlying density set are required.

In the current note, we do not assume compactness conditions but convexity of the density set $\mathcal{F}$; that assumption is realistic for many density classes used in practice. Also, our consideration include estimation based on indirect data $\left(\Phi_{d} \neq \mathrm{Id}\right)$ and estimation for general norms $\|\cdot\|_{T}$.

Considering convex parameter spaces is common in linear functional estimation, see Ibragimov and Has'minskii (1984), Donoho (1994), Cai and Low (2004); where the condition of convexity is critically addressed in the latter note.

As the main result (Section 2), we show equivalence of the individual and uniform optimal rates for convex density sets $\mathcal{F}$ which are Baire spaces with respect to the $L_{1}\left(\mathbb{R}^{d}\right)$-metric, by using an appropriate randomized estimator $\hat{f}_{n}$. Under slight additional assumptions, that equivalence is kept for non-randomized estimators. We will see that the techniques of our proof do not require usual compactness conditions on $\mathcal{F}$ (finiteness of some covering numbers) but the following condition, which is satisfied by any Baire space (with respect to the $L_{1}\left(\mathbb{R}^{d}\right)$-norm), is sufficient: 


\section{Condition A}

If $\mathcal{F}$ is equal to the union of countably many $\mathcal{F}$-closed subsets $A_{k} \subseteq \mathcal{F}, k \in \mathbb{N}$, with respect to the $L_{1}\left(\mathbb{R}^{d}\right)$-topology, then at least one of the $A_{k}$ contains a non-empty, open ball in $\mathcal{F}$ with respect to the $L_{1}\left(\mathbb{R}^{d}\right)$-norm.

One can show that Condition $\mathrm{A}$ is satisfied by any $L_{1}\left(\mathbb{R}^{d}\right)$-closed density set $\mathcal{F}$. That follows immediately from Baire's theorem (e.g. Yosida (1980, p. 11 f)). Furthermore, every non-empty locally compact Hausdorff space (with respect to the $L_{1}\left(\mathbb{R}^{d}\right)$-metric) is a Baire space and, hence, fulfills Condition A. For the underlying topological theory, see for instance Munkres (2000) or the original work of Baire (1899).

Also, we will give a counterexample of that equivalence for some convex set $\mathcal{F}$ which is not a Baire set. Applications are discussed in Section 3; the proofs are deferred to Section 4.

\section{Main results}

As a necessary condition, we assume continuity of $\Phi_{t}$ on $\mathcal{F}$. Otherwise there is no uniformly consistent estimator at all so that (1.2) cannot be satisfied, see the following Lemma 2.1, part (a).

Further, we assume that $\left\|\Phi_{t}\right\|:=\sup _{f \in \mathcal{F}}\left\|\Phi_{t}(f)\right\|_{T}<\infty$. We mention that those conditions follow immediately if continuity of $\Phi_{t}$ is not only assumed on $\mathcal{F}$ but on the whole domain $F$. Without loss of generality, we may assume that $\left\|\hat{f}_{n}\right\|_{T} \leq\left\|\Phi_{t}\right\|$ almost surely; since, $\Gamma_{n}(f)$ is multiplied by factor $2^{k}$, at most, when changing $\hat{f}_{n}$ into the truncated estimator $\left\|\Phi_{t}\right\| \hat{f}_{n} / \max \left\{\left\|\Phi_{t}\right\|,\left\|\hat{f}_{n}\right\|_{T}\right\}$. Therefore, the convergence rates are not affected.

Lemma 2.1 Assume that $\Phi_{d}$ is continuous on $\mathcal{F}$; and that $\Phi_{d}(f)$ is a density for any $f \in \mathcal{F} ;$ further, $\left\|\Phi_{t}\right\|<\infty$. Then,

(a) if $\Phi_{t}$ - restricted to $\mathcal{F}$ - is discontinuous at at least one $f \in \mathcal{F}$ then we have $\sup _{f \in \mathcal{F}} \Gamma_{n}(f) \stackrel{n \rightarrow \infty}{\longrightarrow} \infty$ for any estimator $\hat{f}_{n}$ and any sequence $\left(a_{n}\right)_{n} \downarrow 0$,

(b) if, in addition, $\Phi_{t}$ is continuous on $\mathcal{F}$, then each $\Gamma_{n}$ is continuous on $\mathcal{F}$ with respect to the $L_{1}\left(\mathbb{R}^{d}\right)$-topology.

Therefore, each $\Gamma_{n}$ is a non-linear and continuous functional mapping $\mathcal{F}$ to $[0, \infty)$. We give

Theorem 2.2 Assume that $\Phi_{d}$ and $\Phi_{t}$ are linear on $F$ and continuous on $\mathcal{F}$ with respect to $\left(L_{1}\left(\mathbb{R}^{d}\right), L_{1}\left(\mathbb{R}^{d}\right)\right)$ and $\left(L_{1}\left(\mathbb{R}^{d}\right),\|\cdot\|_{T}\right)$, respectively; that $\left\|\Phi_{t}\right\|<\infty$; that all $\Phi_{d}(f)$ with $f \in \mathcal{F}$ are densities; that $\mathcal{F}$ is convex; and Condition $A$ is satisfied.

(a) If, for some estimator $\hat{f}_{n},(1.1)$ is satisfied, then (1.2) is valid for some randomized estimator $\tilde{f}_{n}$ for the same $\left(a_{n}\right)_{n}$. 
(b) If, in addition, $\left(T,\|\cdot\|_{T}\right)$ is either a reflexive separable Banach space or equal to $L_{1}\left(\mathbb{R}^{d}\right)$, then (1.2) holds true for some non-randomized estimator $\tilde{f}_{n}$ for the same $\left(a_{n}\right)_{n}$, too.

Hence, we have shown coincidence of the optimal uniform and individual rates for any convex Baire space $\mathcal{F}$. The conditions of part (b) of the theorem are satisfied by $T=L_{2}\left(\mathbb{R}^{d}\right)$, for example.

Nevertheless, we show that Theorem 2.2 does not hold for a general convex set $\mathcal{F}$ when Condition A is violated by giving an explicit univariate $(d=1)$ counterexample. Consider the smoothness class

$$
\mathcal{F}:=\left\{f \text { density : } f^{f t} \text { is compactly supported and } \sup _{t \in \mathbb{R}}\left(1+|t|^{\beta+1 / 2}\right)\left|f^{f t}(t)\right| \leq C\right\},
$$

for some fixed $C>0$ and $\beta>1 / 2$, where $f^{f t}$ denotes the Fourier transform of a function $f$, defined by

$$
f^{f t}(t)=\int \exp (i\langle t, x\rangle) f(x) d x
$$

in the general multivariate case; and we write $\langle\cdot, \cdot\rangle$ for the standard inner product in $\mathbb{R}^{d}$. In the current setting, the inner product reduces to the product of real numbers. We see that $\mathcal{F}$ is convex. Put $\Phi_{d}=\Phi_{t}=\mathrm{Id} ; T=L_{2}(\mathbb{R})$ with the corresponding norm; and $k=2$. Then, the standard kernel estimator with the sinc-kernel $K(x)=\sin (x) /(\pi x)$ satisfies (1.1) for $a_{n}=n^{-1} h_{n}^{-1}$ where any sequence tending to zero is eligible for the bandwidth $h=h_{n}$. That can be seen by using the standard methods introduced by Watson and Leadbetter (1963).

On the other hand, there is no estimator satisfying (1.2) for $a_{n} n^{2 \beta /(2 \beta+1)} \stackrel{n \rightarrow \infty}{\longrightarrow} 0$ when $\mathcal{F}$ is as in (2.1). That can be seen as follows: First we define the densities $K_{\omega}(x)=$ $(1-\cos (\omega x)) /\left(\pi \omega x^{2}\right)$ having the Fourier transform $K_{\omega}^{f t}(t)=(1-|t / \omega|)_{+}$. We introduce

$$
\mathcal{F}^{\prime}:=\left\{f \text { density }: \sup _{t \in \mathbb{R}}\left(1+|t|^{\beta+1 / 2}\right)\left|f^{f t}(t)\right| \leq C\right\} .
$$

As $\left\{K_{\omega} * f: \omega>0, f \in \mathcal{F}^{\prime}\right\} \subseteq \mathcal{F}$ and $\left\|K_{\omega} * f-f\right\|_{L_{1}(\mathbb{R})} \stackrel{\omega \rightarrow \infty}{\longrightarrow} 0$ for any $f \in L_{1}(\mathbb{R})$, the set $\mathcal{F}$ lies dense in $\mathcal{F}^{\prime}$. Since $\sup \left\{\|f\|_{T}: f \in \mathcal{F}^{\prime}\right\}<\infty$ and the $L_{2}(\mathbb{R})$-convergence is dominated by the $L_{1}\left(\mathbb{R}\right.$ )-convergence on $\mathcal{F}^{\prime}$ (as we will see in Subsection 3.1 in a broader context) $\Gamma_{n}$ is $L_{1}(\mathbb{R})$-continuous on $\mathcal{F}^{\prime}$ according to Lemma 2.1(b). Combining those two facts, we obtain for any sequence $\left(a_{n}\right)_{n}$ and all integers $n>0$ that

$$
\sup _{f \in \mathcal{F}} \Gamma_{n}(f)=\sup _{f \in \mathcal{F}^{\prime}} \Gamma_{n}(f) \text {. }
$$

It is a well-known fact that $\sup _{f \in \mathcal{F}^{\prime}} \Gamma_{n}(f)$ is bounded below when setting $a_{n}=$ $n^{-2 \beta /(2 \beta+1)}$, e.g. Stone (1980), Donoho and Liu (1991a, b), Hall and Meister (2007).

While any rate arbitrarily close to $n^{-1}$ - also known as the parametric rate - is achievable as the individual rate, the optimal uniform rate corresponds to the classical minimax rate for smoothness classes. Hence, the individual and the uniform rate are different from each other. 
The fact that Condition $\mathrm{A}$ is violated and, hence, $\mathcal{F}$ is not a Baire space can also be understood directly: Note that $\mathcal{F}$ may be represented by a countable union of those sets $\mathcal{F}_{N}$ containing all $f \in \mathcal{F}$ whose Fourier transforms are supported on $[-N, N]$. As $L_{1}(\mathbb{R})$-convergence implies uniform convergence in the Fourier domain we realize that all $\mathcal{F}_{N}$ are $L_{1}(\mathbb{R})$-closed. On the other hand, none of the $\mathcal{F}_{N}$ contains an $L_{1}(\mathbb{R})$-open ball of $\mathcal{F}$ as any $f \in \mathcal{F}_{N}$ may be approximated in the $L_{1}(\mathbb{R})$-norm by the sequence $\left(f_{n}\right)_{n}$ with $f_{n}=(1 / n) g+(1-1 / n) f$ where $g \in \mathcal{F} \backslash \mathcal{F}_{N}$ is fixed.

\section{Applications and discussion}

\subsection{Standard density estimation}

$\Phi_{d}=\Phi_{t}=\mathrm{Id}, \mathcal{F} \subseteq T$. When putting $\|\cdot\|_{T}$ equal to the $L_{1}\left(\mathbb{R}^{d}\right)$-norm, Theorem 2.2 says that equivalence for individual and uniform rates occurs whenever $\mathcal{F}$ is a convex $L_{1}\left(\mathbb{R}^{d}\right)$ Baire space; for another norm $\|\cdot\|_{T}$, the $L_{2}\left(\mathbb{R}^{d}\right)$-norm, for instance, we have to assume that convergence with respect to that norm is dominated by the $L_{1}\left(\mathbb{R}^{d}\right)$-convergence on $\mathcal{F}$, i.e. $\left\|f_{n}-f\right\|_{L_{1}(\mathbb{R})} \stackrel{n \rightarrow \infty}{\longrightarrow} 0 \Longrightarrow\left\|f_{n}-f\right\|_{T} \stackrel{n \rightarrow \infty}{\longrightarrow} 0$ for any sequence $\left(f_{n}\right)_{n}$ in $\mathcal{F}$ and $f \in \mathcal{F}$; and we have to ensure that $\mathcal{F}$ is uniformly bounded above with respect to $\|\cdot\|_{T}$.

As important examples for $\mathcal{F}$, we consider the smoothness classes

$$
\mathcal{F}:=\left\{f \text { density }: \sup _{t \in \mathbb{R}^{d}} \varphi(t)\left|f^{f t}(t)\right| \leq C\right\},
$$

with fixed constant $C>0$ and some positive-valued continuous function $\varphi$; usually, $\varphi \sim\|t\|^{\beta+d-1 / 2}$ for some $\beta>1 / 2$ (also see (2.2)); but also exponentially increasing functions $\varphi$ are of practical interest leading to so-called supersmooth densities. All those classes satisfy the conditions of Theorem 2.2; convexity is rather elementary and the Baire space criterion is shown by $L_{1}\left(\mathbb{R}^{d}\right)$-closedness as follows: Assume some sequence $\left(f_{n}\right)_{n} \subseteq \mathcal{F}$ converging to some $f \in L_{1}\left(\mathbb{R}^{d}\right)$. Clearly, $f$ is a density and, for any $t \in \mathbb{R}^{d}$,

$$
\left|f^{f t}(t)\right| \leq\left|f_{n}^{f t}(t)\right|+\left|f_{n}^{f t}(t)-f^{f t}(t)\right| \leq C / \varphi(t)+\left\|f-f_{n}\right\|_{L_{1}\left(\mathbb{R}^{d}\right)} \stackrel{n \rightarrow \infty}{\longrightarrow} C / \varphi(t),
$$

from what follows that $f \in \mathcal{F}$. Then, defining $\left(T,\|\cdot\|_{T}\right)=\left(L_{p}\left(\mathbb{R}^{d}\right),\|\cdot\|_{L_{p}\left(\mathbb{R}^{d}\right)}\right)$ for $p \in\{1,2\}$, we verify that $\Phi_{t}=$ Id is a continuous operator $\mathcal{F} \rightarrow T$ and that $\sup _{f \in \mathcal{F}}\|f\|_{T}<\infty$; this result is obvious for $p=1$; while, in the case of $p=2$, we have to use Parseval's identity and the fact that $L_{1}\left(\mathbb{R}^{d}\right)$-convergence implies uniform convergence in the Fourier domain; also, we assume that $1 / \varphi^{2}$ is integrable on the whole real line. More concretely, we have

$$
\begin{aligned}
(2 \pi)^{d}\|f-\tilde{f}\|_{L_{2}\left(\mathbb{R}^{d}\right)}^{2} & =\int\left|f^{f t}(t)-\tilde{f}^{f t}(t)\right|^{2} d t \\
& \leq(2 R)^{d} \cdot \sup _{t \in \mathbb{R}^{d}}\left|f^{f t}(t)-\tilde{f}^{f t}(t)\right|^{2}+4 \int_{|t|>R}(\varphi(t))^{-2} d t \\
& \leq(2 R)^{d} \cdot\|f-\tilde{f}\|_{L_{1}\left(\mathbb{R}^{d}\right)}^{2}+4 \int_{|t|>R}(\varphi(t))^{-2} d t
\end{aligned}
$$


for any $R>0$. Then, continuity of $\Phi_{t}$ on $\mathcal{F}$ follows by standard arguments. Uniform boundedness of $\|f\|_{T}$ for $f \in \mathcal{F}$ is implied putting $\tilde{f}=0$ in the above inequality. As the linear space $F$, we may select $F=L_{1}\left(\mathbb{R}^{d}\right) \cap L_{2}\left(\mathbb{R}^{d}\right)$.

To study the pointwise rates at some $x \in \mathbb{R}^{d}$ for those smoothness classes $\mathcal{F}$, we put $\Phi_{t}(f)=f(x)=(2 \pi)^{-d} \int \exp (-i\langle t, x\rangle) f^{f t}(t) d t$. Continuity of $\Phi_{t}$ and $\left\|\Phi_{t}\right\|<\infty$ occur whenever $\varphi^{-1}$ is integrable on $\mathbb{R}^{d}$.

Therefore, we have shown coincidence of the optimal uniform and individual rates under common conditions in density estimation.

\subsection{Density deconvolution}

As mentioned in the introduction, we put $\Phi_{d}(f)=g * f$, i.e. the convolution of $g$ and $f$. As $g$ is a density function, we have $\left\|\Phi_{d}(f)\right\|_{L_{p}\left(\mathbb{R}^{d}\right)} \leq\|f\|_{L_{p}\left(\mathbb{R}^{d}\right)}$ for $p \in\{1,2\}$ and all $f \in L_{1}\left(\mathbb{R}^{d}\right) \cap L_{2}\left(\mathbb{R}^{d}\right)$. Therefore, the setting of the error-free case 3.1 can be adopted. While optimal uniform rates have been established (e.g. Fan (1991, 1993), Hall and Meister (2007)), those rates may be extended to the individual case by Theorem 2.2. To our knowledge, individual rates in this deconvolution problem have not been considered before.

\subsection{Nonparametric regression}

Assume a regression experiment where the i.i.d. data $\left(X_{1}, Y_{1}\right), \ldots,\left(X_{n}, Y_{n}\right)$ having the density $f_{(X, Y)}(x, y)$ are observed. We assume that there is some $S>0$ with $f_{(X, Y)}(x, y)=0, \forall|y|>S, \forall f_{(X, Y)} \in \mathcal{F}$. In practice, this means that the regression function and the support of the regression error distribution are uniformly bounded. Further, we put $\Phi_{d}=\mathrm{Id}, \Phi_{t}\left(f_{(X, Y)}\right)=\left(\int f_{(X, Y)}(x, y) d y, \int y f_{(X, Y)}(x, y) d y\right),\|t\|_{T}=$ $\int\left(\left|t_{1}(x)\right|+\left|t_{2}(x)\right|\right) d x$. Therefore, the first component represents the design density; while the second component shall be interpreted as the product of the design density and the regression function. We can verify that $\Phi_{t}$ is continuous and bounded on $\mathcal{F}$ with respect to the $L_{1}\left(\mathbb{R}^{d+1}\right)$-norm on $\mathcal{F}$. Whenever $\mathcal{F}$ is a convex $L_{1}\left(\mathbb{R}^{d+1}\right)$-Baire space Theorem 2.2 can be applied.

\section{Proofs}

Proof of Lemma 2.1: (a) By assumption, there is some sequence $\left(f_{n}\right)_{n} \subseteq \mathcal{F}$ with $\left\|f-f_{n}\right\|_{L_{1}\left(\mathbb{R}^{d}\right)} \stackrel{n \rightarrow \infty}{\longrightarrow} 0$ and $\inf _{n}\left\|\Phi_{t}(f)-\Phi_{t}\left(f_{n}\right)\right\|_{T}>0$. Then, we have

$$
\begin{aligned}
& 2 \sup _{f^{\prime} \in \mathcal{F}} \Gamma_{n}\left(f^{\prime}\right) \geq \Gamma_{n}(f)+\Gamma_{n}\left(f_{n}\right) \\
& f^{\prime} \in \mathcal{F} \\
& =a_{n}^{-1} \int \ldots \int \mid\left\|\hat{f}_{n}\left(x_{1}, \ldots, x_{n}\right)-\Phi_{t}(f)\right\|_{T}^{k} \prod_{j=1}^{n}\left[\Phi_{d}(f)\right]\left(x_{j}\right) \\
& +\left\|\hat{f}_{n}\left(x_{1}, \ldots, x_{n}\right)-\Phi_{t}\left(f_{n}\right)\right\|_{T}^{k} \prod_{j=1}^{n}\left[\Phi_{d}\left(f_{n}\right)\right]\left(x_{j}\right) \mid d x_{1} \cdots d x_{n}
\end{aligned}
$$




$$
\begin{aligned}
\geq a_{n}^{-1} \int \ldots \int & \left|\left\|\hat{f}_{n}\left(x_{1}, \ldots, x_{n}\right)-\Phi_{t}(f)\right\|_{T}^{k}+\left\|\hat{f}_{n}\left(x_{1}, \ldots, x_{n}\right)-\Phi_{t}\left(f_{n}\right)\right\|_{T}^{k}\right| \\
& \prod_{j=1}^{n}\left[\Phi_{d}(f)\right]\left(x_{j}\right) d x_{1} \cdots d x_{n} \\
& -a_{n}^{-1} \int \ldots \mid\left\|\hat{f}_{n}\left(x_{1}, \ldots, x_{n}\right)-\Phi_{t}\left(f_{n}\right)\right\|_{T}^{k} \\
& \left|\prod_{j=1}^{n}\left[\Phi_{d}(f)\right]\left(x_{j}\right)-\prod_{j=1}^{n}\left[\Phi_{d}\left(f_{n}\right)\right]\left(x_{j}\right)\right| d x_{1} \cdots d x_{n} \\
\geq & a_{n}^{-1} 2^{1-k}\left\|\Phi_{t}(f)-\Phi_{t}\left(f_{n}\right)\right\|_{T}^{k}-a_{n}^{-1}\left(2\left\|\Phi_{t}\right\|\right)^{k} n\left\|\Phi_{d}(f)-\Phi_{d}\left(f_{n}\right)\right\|_{L_{1}\left(\mathbb{R}^{d}\right)} .
\end{aligned}
$$

By replacing $\left(f_{n}\right)_{n}$ by an appropriate subsequence we are able to guarantee that $n \| \Phi_{d}(f)$ $-\Phi_{d}\left(f_{n}\right) \|_{L_{1}\left(\mathbb{R}^{d}\right)} \stackrel{n \rightarrow \infty}{\longrightarrow} 0$ so that $\sup _{f^{\prime} \in \mathcal{F}} \Gamma_{n}\left(f^{\prime}\right)$ tends to infinity as $n \rightarrow \infty$.

(b) We have for any $f, f^{\prime} \in \mathcal{F}$,

$$
\begin{aligned}
& \left|\Gamma_{n}(f)-\Gamma_{n}\left(f^{\prime}\right)\right| \\
& \leq a_{n}^{-1} \int \ldots \int \mid\left\|\hat{f}_{n}\left(x_{1}, \ldots, x_{n}\right)-\Phi_{t}(f)\right\|_{T}^{k} \prod_{j=1}^{n}\left[\Phi_{d}(f)\right]\left(x_{j}\right) \\
& \quad-\left\|\hat{f}_{n}\left(x_{1}, \ldots, x_{n}\right)-\Phi_{t}\left(f^{\prime}\right)\right\|_{T}^{k} \prod_{j=1}^{n}\left[\Phi_{d}\left(f^{\prime}\right)\right]\left(x_{j}\right) \mid d x_{1} \cdots d x_{n} \\
& \quad a_{n}^{-1} \int \ldots \int \mid\left\|\hat{f}_{n}\left(x_{1}, \ldots, x_{n}\right)-\Phi_{t}(f)\right\|_{T}^{k} \\
& \quad-\left\|\hat{f}_{n}\left(x_{1}, \ldots, x_{n}\right)-\Phi_{t}\left(f^{\prime}\right)\right\|_{T}^{k} \mid \prod_{j=1}^{n}\left[\Phi_{d}(f)\right]\left(x_{j}\right) d x_{1} \cdots d x_{n} \\
& \quad+a_{n}^{-1} \int \ldots \int_{j}^{n}\left\|\hat{f}_{n}\left(x_{1}, \ldots, x_{n}\right)-\Phi_{t}\left(f^{\prime}\right)\right\|_{T}^{k} \\
& \qquad\left|\prod_{j=1}^{n}\left[\Phi_{d}(f)\right]\left(x_{j}\right)-\prod_{j=1}^{n}\left[\Phi_{d}\left(f^{\prime}\right)\right]\left(x_{j}\right)\right| d x_{1} \cdots d x_{n} \\
& \leq a_{n}^{-1} k\left(2\left\|\Phi_{t}\right\|\right)^{k-1}\left\|\Phi_{t}(f)-\Phi_{t}\left(f^{\prime}\right)\right\|_{T}+a_{n}^{-1}\left(2\left\|\Phi_{t}\right\|\right)^{k} n\left\|\Phi_{d}(f)-\Phi_{d}\left(f^{\prime}\right)\right\|_{L_{1}\left(\mathbb{R}^{d}\right)},
\end{aligned}
$$

so that continuity of $\Phi_{t}$ and $\Phi_{d}$ is applicable.

Proof of Theorem 2.2: (a) Assume (1.1) with respect to some known $\left(a_{n}\right)_{n}$ and some estimator $\hat{f}_{n}$. Hence, we have boundedness of $\left\{\Gamma_{n}(f): n \in \mathbb{N}\right\}$ for each $f \in \mathcal{F}$. Applying Lemma 2.1(b), we notice that $\left\{\Gamma_{n}: n \in \mathbb{N}\right\} \subseteq C(\mathcal{F}, \mathbb{R})$, denoting the set of all continuous mappings from $\mathcal{F}$ to $\mathbb{R}$. Put

$$
C_{m}=\bigcap_{n \in \mathbb{N}}\left\{f \in \mathcal{F}: \Gamma_{n}(f) \leq m\right\},
$$


for any positive integer $m$. We notice that $C_{m}$ is $\mathcal{F}$-closed due to the continuity of all $\Gamma_{n}$. It follows from (1.1) that $\mathcal{F}=\bigcup_{m \in \mathbb{N}} C_{m}$. Based on Condition A, we conclude that there exist a positive constant $C>0$ and an open ball $B_{\varepsilon}\left(f_{0}\right)=\left\{f \in \mathcal{F}:\left\|f-f_{0}\right\|_{L_{1}\left(\mathbb{R}^{d}\right)}<\varepsilon\right\}$, $\varepsilon>0, f_{0} \in \mathcal{F}$, where

$$
\sup _{f \in B_{\varepsilon}\left(f_{0}\right)} \sup _{n \in \mathbb{N}} \Gamma_{n}(f) \leq C
$$

That reasoning is part of the proof of the famous uniform boundedness principle in functional analysis; however, as $\Gamma_{n}$ is not a linear operator, we must not apply this theorem in its common version.

As $\hat{f}_{n}, \mathcal{F}, \Phi_{d}, \Phi_{t}$ and $\left(a_{n}\right)_{n}$ are known we also (theoretically) know some $\varepsilon$ and $f_{0}$ so that (4.1) is satisfied. Note that $\varepsilon>0$ may be seen as sufficiently small; in particular, we may assume that $\varepsilon<3$. Therefore, those quantities may be used to construct new estimators.

To extend (4.1) to the whole of $\mathcal{F}$, we introduce the mapping

$$
P(f)=\frac{\varepsilon}{3} f+\left(1-\frac{\varepsilon}{3}\right) f_{0} .
$$

Due to the convexity of $\mathcal{F}$, we have $P(f) \in \mathcal{F}$ for any $f \in \mathcal{F}$. Further, consider

$$
\left\|P(f)-f_{0}\right\|_{L_{1}\left(\mathbb{R}^{d}\right)}=\frac{\varepsilon}{3}\left\|f-f_{0}\right\|_{L_{1}\left(\mathbb{R}^{d}\right)}<\varepsilon,
$$

from what follows that all $P(f)$ with $f \in \mathcal{F}$ are contained in $B_{\varepsilon}\left(f_{0}\right)$. Independently of the data $X_{1}, \ldots, X_{n}$, we generate i.i.d. Bernoulli random variables $\delta_{1}, \ldots, \delta_{n}$ with $P\left[\delta_{1}=1\right]=\varepsilon / 3$ and i.i.d. random variables $Y_{1}, \ldots, Y_{n}$ having the density $\Phi_{d}\left(f_{0}\right)$. Define

$$
X_{j}^{*}=\delta_{j} X_{j}+\left(1-\delta_{j}\right) Y_{j}, \quad j=1, \ldots, n .
$$

Hence, the $X_{j}^{*}$ are i.i.d. and have the density $\Phi_{d}(P(f))$. That inspires us to employ

$$
\tilde{f}_{n}\left(X_{1}, \ldots, X_{n}\right):=\frac{3}{\varepsilon} \hat{f}_{n}\left(X_{1}^{*}, \ldots, X_{n}^{*}\right)+\frac{\varepsilon-3}{\varepsilon}\left[\Phi_{t}\left(f_{0}\right)\right]
$$

as the estimator for $\Phi_{t}(f), f \in \mathcal{F}$, where $\hat{f}_{n}$ is the estimator taken from $\Gamma_{n}$. Then,

$$
\begin{aligned}
\sup _{f \in \mathcal{F}} E\left\|\tilde{f}_{n}\left(X_{1}, \ldots, X_{n}\right)-\Phi_{t}(f)\right\|_{T}^{k} & =\left(\frac{3}{\varepsilon}\right)^{k} \sup _{f \in \mathcal{F}} E\left\|\hat{f}_{n}\left(X_{1}^{*}, \ldots, X_{n}^{*}\right)-\Phi_{t}(P(f))\right\|_{T}^{k} \\
& \leq\left(\frac{3}{\varepsilon}\right)^{k} \sup _{f \in B_{\varepsilon}\left(f_{0}\right)} E\left\|\hat{f}_{n}\left(X_{1}, \ldots, X_{n}\right)-\Phi_{t}(f)\right\|_{T}^{k} \\
& =O\left(a_{n}\right),
\end{aligned}
$$

where (4.1) is used in the latter step. Then, by inserting estimator $\tilde{f}_{n}$ for $\hat{f}_{n}$ in $\Gamma_{n}$, both (1.1) and (1.2) hold.

(b) If $T$ is either a reflexive separable Banach space or equal to $L_{1}\left(\mathbb{R}^{d}\right)$, then the following conditional expectation

$$
\tilde{f}_{n ; \text { det. }}\left(X_{1}, \ldots, X_{n}\right)=E\left(\tilde{f}_{n}\left(X_{1}, \ldots, X_{n}\right) \mid X_{1}, \ldots, X_{n}\right)
$$


is well-defined, known and satisfies

$$
\begin{aligned}
& E\left\|\tilde{f}_{n ; \text { det. }}\left(X_{1}, \ldots, X_{n}\right)-\Phi_{t}(f)\right\|_{T}^{k} \\
& \quad=E\left\|E\left(\tilde{f}_{n}\left(X_{1}, \ldots, X_{n}\right)-\Phi_{t}(f) \mid X_{1}, \ldots, X_{n}\right)\right\|_{T}^{k} \\
& \quad \leq E E\left(\left\|\tilde{f}_{n}\left(X_{1}, \ldots, X_{n}\right)-\Phi_{t}(f)\right\|_{T}^{k} \mid X_{1}, \ldots, X_{n}\right) \\
& \quad=E\left\|\tilde{f}_{n}\left(X_{1}, \ldots, X_{n}\right)-\Phi_{t}(f)\right\|_{T}^{k},
\end{aligned}
$$

where Jensen's inequality (note that $k \geq 1$ ), along with the general inequality $\|E Z\|_{T} \leq$ $E\|Z\|_{T}$ for any integrable random variable $Z$ taking its values in $T$, have been used. That latter inequality is rather elementary if $T=L_{1}\left(\mathbb{R}^{d}\right)$; for a general reflexive separable Banach space, see the book of Ledoux and Talagrand (1991) for the theory of integration over separable and reflexive Banach spaces. Finally, we realize that $\tilde{f}_{n \text {; det }}\left(X_{1}, \ldots, X_{n}\right)$ is a non-randomized estimator, which is calculable since the distributions of the $\delta_{j}$ and $Y_{j}$ are known. Then, (4.2) proves that the risk of estimator $\tilde{f}_{n ; \text { det. }}\left(X_{1}, \ldots, X_{n}\right)$ is smaller or equal to that of the randomized estimator $\tilde{f}_{n}\left(X_{1}, \ldots, X_{n}\right)$ as defined in the proof of part (a) for all $f \in \mathcal{F}$. Hence, combining (4.2) with part (a) of the theorem, the assertion of part (b) holds true when using the estimator $\tilde{f}_{n \text {; det. }}\left(X_{1}, \ldots, X_{n}\right)$ instead of $\tilde{f}_{n}\left(X_{1}, \ldots, X_{n}\right)$.

Acknowledgments. The author is grateful to the Associate Editor for valuable comments on his work.

\section{References}

[1] Antos, A., Lower bounds for the rate of convergence in nonparametric pattern recognition. Theo. Comp. Science 284 (2002), pp. 3-24.

[2] Antos, A., Györfi, L. and Kohler, M., Lower bounds on the rate of convergence of nonparametric regression estimates. J. Statist. Plann. Inf. 83 (2000), pp. 91-100.

[3] Antos, A. and Lugosi, G., Strong minimax lower bounds for learning. Machine Learning 30 (1998), pp. 31-56.

[4] Baire, R.-L., Sur les fonctions de variables réelles. Annali di Mat. Ser. 3 , 3 (1899), pp. 1-123.

[5] Barron, A. and Hengartner, N., Information theory and superefficiency. Ann. Statist. 26 (1998), pp. 1800-1825.

[6] Birgé, L., On estimating a density using Hellinger distance and some other strange facts. Prob. Theo. Rel. Fields 7 (1986), pp. 271-291.

[7] Brown, L. D., Low, M. G. and Zhao, L. H., Superefficiency in nonparametric function estimation. Ann. Statist. 25 (1997), pp. 2607-2625.

[8] Cai, T. and Low, M. G., Minimax estimation of linear functionals over nonconvex parameter spaces. Ann. Statist. 32 (2004), pp. 552-576. 
[9] Devroye, L., On arbitrarily slow rates of global convergence in density estimation. Z. Wahrsch. Verw. Gebiete 71 (1983), pp. 271-291.

[10] Devroye, L., Another proof of a slow convergence result of Birgé. Statist. Probab. Lett. 23 (1995), pp. 63-67.

[11] Donoho, D. L., Statistical estimation and optimal recovery. Ann. Statist. 22 (1994), pp. 238-270.

[12] Donoho, D. L. and Liu, R. C., Geometrizing rates of convergence II. Ann. Statist. 19 (1991a), pp. 633-667.

[13] Donoho, D. L. and Liu, R. C., Geometrizing rates of convergence III. Ann. Statist. 19 (1991b), pp. 668-701.

[14] Fan, J., On the optimal rates of convergence for nonparametric deconvolution problems. Ann. Statist. 19 (1991), pp. 1257-1272.

[15] Fan, J., Adaptively local one-dimensional subproblems with application to a deconvolution problem. Ann. Statist. 21 (1993), pp. 600-610.

[16] Ibragimov, I. A. and Has'minskii, R. Z., Nonparametric estimation of the value of a linear functional in Gaussian white noise. Theo. Probab. Appl. 29 (1984), pp. 18-32.

[17] Hall, P. and Meister, A., A ridge-parameter approach to deconvolution. Ann. Statist. 35 (2007), pp. 1535-1558.

[18] Korostelev. A. P. and Tsybakov, A. B., Minimax Theory of Image Reconstruction, Springer, 1993.

[19] Ledoux, M. and Talagrand, M., Probability in Banach Spaces. Springer, 1991.

[20] Munkres, J., Topology, 2nd ed., Prentice Hall, 2000.

[21] Stone, C. J., Optimal rates of convergence for nonparametric estimators. Ann. Statist. 8 (1980), pp. 1348-1360.

[22] Watson, G. S. and Leadbetter, M. R., On the estimation of the probability density, I. Ann. Math. Statist. 34 (1963), pp. 480-491.

[23] Yang, Y. and Barron, A., Information-theoretic determination of minimax rates of convergence. Ann. Statist. 27 (1999), pp. 1564-1599.

[24] Yosida, K., Functional Analysis. 6th ed., Springer, 1980.

\author{
Alexander Meister \\ Graduiertenkolleg 1100 \\ Universität Ulm \\ Helmholtzstraße 22 \\ 89081 Ulm \\ Germany \\ alexander.meister@uni-ulm.de
}

